\title{
Study on the Sensitivity Analysis of Cost Index:A Case of Flue-cured Tobacco Production
}

\author{
Dongbing Huang 1, a, Yin Wang ${ }^{2, b}$,Zehua Ying ${ }^{3, c}$ and Junfang Liü,d \\ ${ }^{1}$ Guizhou University of Finance and economics, Guizhou, 550025, China \\ ${ }^{2}$ Guizhou University of Finance and economics, Guizhou, 550025, China \\ ${ }^{3}$ Guizhou University of Finance and economics, Guizhou, 550025, China \\ ${ }^{4}$ Guizhou University of Finance and economics, Guizhou, 550025, China \\ ahuangdongbing@mail.gufe.edu.cn, b1107070983@qq.com, c981031582@qq.com, \\ d825633379@qq.com
}

\begin{abstract}
Keywords: Sensitivity analysis; cost index; tobacco production
Abstract. To effectively manage and control the cost of flue-cured tobacco has a strong theoretical significance and practical guidance. Firstly, the concept of the sensitivity of tobacco production cost index is introduced, and the sensitivity analysis model of tobacco production cost index is established. Secondly, the model is used to calculate the elasticity of each variable of flue-cured tobacco production cost. The effectiveness of the method is proved by a case study. Finally, how to use this model to control production cost of flue-cured tobacco is discussed.
\end{abstract}

\section{Introduction}

In general, the tobacco production has the following characteristics. There are many cost items, including material and service costs, labor costs, land costs, etc. The production cycle is relatively long, generally for nine months. Except the process of breeding, cultivated land, ridging, pesticide and fertilization of crops, there is a most important process which is redrying process.

The concept of sensitivities of production cost index of flue-cured tobacco was introduced by using the theory of sensitivity analysis. And the tobacco production cost index sensitivity analysis model is established. So it has a strong theoretical and practical significance for the farmers to effectively manage and control the cost of tobacco.

\section{The Sensitivity of Production Cost Index of Flue-cured Tobacco}

Sensitivity analysis is a commonly used method of uncertainty analysis. Firstly, it extracts the sensitive factors from many uncertain factors, and then studies the influence degree of the change of the uncertain factors to the evaluation index [1]. Many factors affecting the objectives of the project are often uncertain changes. In order to achieve the purpose of decision-making, we need to measure and analyze the influence degree of the change of one or more uncertain factors to the cost goal. The hypothetical model is $\mathrm{y}=f\left(x_{1}, x_{2}, \mathrm{~K}, x_{n}\right)$.Here, $x_{i}$ represents the $\mathrm{ith}(\mathrm{i}=1,2 \ldots \mathrm{n})$ influencing factor, and each attribute value is in the range of possible changes and predict the impact of these factors on the output value y of the model [2].

The main goal of the sensitivity analysis is to analyze the most sensitive factors and their sensitivity. Usually a single factor change is analyzed. If necessary, the degree of impact of multiple uncertainties can also be analyzed. The sensitivity of the project indicator to the sensitivity factor indicates the change range of the evaluation index is caused by the sensitivity factor according to a certain percentage change [3].

Tobacco production has many cost items, long production cycle and high technical requirements. The influence of various market factors on the cost of flue-cured tobacco production is great. So there are many uncertain factors in the cost change, that is, there are many uncertain factors in the change of cost index. In general, the impact of the cost of tobacco production index uncertainties are the quantity and price of each cost item in base and report period, the amount of labor and labor 
prices in the base period and report period, the per acre price of land in the base period and report period and so on. Through the multi-factor sensitivity analysis of tobacco production cost index, we can find out the sensitive factors and sensitivity of flue-cured tobacco production. So we can grasp the key factors in flue-cured tobacco production process, control the cost effectively, and get a multiplier effect.

\section{Analysis model of tobacco production cost index}

Tobacco production cost index can be expressed as the following function:

$$
K=f\left(c_{1}, c_{2}, \mathrm{~L}, c_{n}\right) .
$$

Here, $\mathrm{K}$ represents the production cost index of flue-cured tobacco, $c_{1}$ represents the first factor influencing the production cost index of flue-cured tobacco, and $\mathrm{n}$ represents $\mathrm{n}$ various factors that affect the production cost index of flue-cured tobacco.

When all factors are changed, the influence factor is changed from $c_{1}, c_{2}, \mathrm{~L}, c_{n}$ to $c_{1}^{\prime}, c_{2}^{\prime}, \mathrm{L}, c_{n}^{\prime}$. These changes are respectively $\Delta c_{1}, \Delta c_{2}, \mathrm{~L}, \Delta c_{n}$, that is, $c_{1}^{\prime}=c_{1}+\Delta c_{1}, \quad c_{2}^{\prime}=c_{2}+\Delta c_{2}, \mathrm{~L}, \quad c_{n}^{\prime}=c_{n}+\Delta c_{n}$. It means tobacco production cost index will change, and $K$ will turn into $K^{\prime}$. The change is $\Delta K$, that is $\Delta K=K^{\prime}-K$. When $|\Delta c|$ is not too big, that is, the impact of the factors change little, we can use the multiple functions Taylor expansion [4]:

$$
\Delta K \approx \frac{\partial f}{\partial c_{1}} \times \Delta_{c_{1}}+\frac{\partial f}{\partial_{c_{2}}} \times \Delta_{c_{2}}+\ldots \ldots+\frac{\partial f}{\partial c_{n}} \times \Delta_{c_{n}} .
$$

Here, $\frac{\partial f}{\partial c_{1}}$ is the partial derivative of $c_{1}$ for exponential $\mathrm{K}, \Delta c_{1}$ is the change of $c_{1}$, and $\Delta K$ is the exponential change caused by all factors.

If only one factor changes, and other factors affect the index remain unchanged, that is $\Delta c_{i} \neq 0, \Delta c_{j}=0, j \neq i$, then the change amount of tobacco production cost index can be expressed as $\Delta K_{i}$. And $\Delta c_{i}$ represents the change in the i-factor, that is ${ }_{\Delta K_{i}}=\frac{\partial f}{\partial c_{i}} \times \Delta_{c_{i}}$ [5].

Then, we can obtain $\Delta K=\Delta K_{1}+\Delta K_{2}+\mathrm{L} \mathrm{L} \Delta K_{n}=\sum_{i=1}^{n} K_{i}$.

\section{A sensitivity analysis method of the Flue-cured Tobacco production cost index}

With the above foundation, the sensitivity of farmers' tobacco production cost index can be analyzed, so that we can determine key factors affecting flue-cured tobacco production cost index.

Now we define the ratio $\left(\frac{\Delta K_{i} / K}{\Delta c_{i} / c_{i}}\right)$ of flue-cured tobacco production cost index changes to factors changes that cause them to change as the sensitivity $B_{i}$ of $K$ to $c_{i}$.

$B_{i}$ represents the ith factor $c_{i}$ changes one percentage, which causes the flue-cured tobacco production cost index to change by $B_{i}$ percentage points. When $B_{i}>0$, it indicates that the change direction of the factor is consistent with that of the flue-cured tobacco production cost index. If $c_{i}$ increases by one percentage point, then $K$ will increase by $B_{i}$ percentage points. If $c_{i}$ decreases by one percentage point, then $K$ will also reduce $K$ percentage points. When $B_{i}<0$, it indicates that the change direction of the factor is opposite to that of the flue-cured tobacco production cost index. If $c_{i}$ increases by one percentage point, then $K$ will reduce by $B_{i}$ percentage points. If $c_{i}$ decreases by one percentage point, $K$ will increase by $B_{i}$ percentage 
point. It illustrates that the influence of $c_{i}$ factor on farmers' tobacco production cost index is increased with increasing $\left|B_{i}\right|$. That is to say, farmers' tobacco production cost index is more sensitive to $c_{i}$. So the sensitive factor $c_{i}$ is the focus of the future cost control of tobacco production [6]. The calculation formula of $\mathrm{B}$ is

$$
B_{i}=\frac{\Delta K_{i} / K}{\Delta c_{i} / c_{i}}=\frac{\left(\frac{\partial f}{\partial c_{i}} \times \Delta c_{i}\right) / K}{\Delta c_{i} / c_{i}}=\frac{\partial f}{\partial c_{i}} \times \frac{c_{i}}{K}
$$

In the various stages of flue-cured tobacco planting cost control, we can calculate the sensitivity $B_{i}$ of each factor, then sort them out from large to small. In this way, we can determine the impact of various factors on the production cost index of flue-cured tobacco. In the future of flue-cured tobacco planting process, the focus on those more sensitive factors can accurately and effectively control the cost of tobacco planting.

\section{Case}

The following case shows how to use the parameters $B_{i}$ and analyses the sensitivity of some factors influencing farmers' tobacco production cost index.

The farmers' tobacco production cost index expression is

$$
\mathrm{K}=\sum_{i=1}^{n} \mathrm{p}_{0 i} \times q_{1 i} / \sum \mathrm{p}_{0 i} \times q_{0 i} \approx \frac{10000 \sum \mathrm{p}_{1 i} \times q_{1 i}}{\operatorname{CPI} \sum \mathrm{p}_{0 i} \times q_{0 i}}
$$

where 0 is the base period, 1 is the reporting period, $p_{0 i}$ is the price of the i's cost item in the base period, $q_{0 i}$ is the quantity of the i's cost item in the base period, $p_{1 i}$ is the price of the i's cost item in the reporting period, $q_{1 i}$ is the quantity of the i's cost item in the reporting period, and $\mathrm{K}$ represents the cost index.

Formulas for calculating the sensitivity of each variable.According to the sensitivity formula of the third section and the formula of the farms' tobacco production cost index in the fourth section, the sensitivity of each factor to the farms' tobacco production cost index can be calculated [7].

(1) Calculate the sensitivity $B_{p_{1 i}}$ of $p_{1 i}$

$$
B_{p_{1 i}}=\left(\frac{\partial K}{\partial_{P_{1 i}}}\right) \times \frac{P_{1 i}}{K} \approx \frac{10000 q_{1 i}}{C P I \sum_{i=1}^{n} p_{0 i} q_{01}} \times \frac{p_{1 i}}{10000 \sum_{i=1}^{n} p_{1 i} q_{1 i} / C P I \sum_{i=1}^{n} p_{0 i} q_{0 i}} \approx \frac{P_{1 i} \times q_{1 i}}{\sum_{i=1}^{n} p_{1 i} q_{1 i}}
$$

By the $p_{1 i}$ sensitivity expression can be seen, $B_{p_{1 i}}$ depends on the $p_{1 i}$ and $q_{1 i}$. The bigger the $p_{1 i} \times q_{1 i}$ is, the bigger the sensitivity $B_{p_{1 i}}$ of the $p_{1 i}$ is. And the smaller the $\sum_{i=1}^{n} p_{1 i} q_{1 i}$ is, the bigger the sensitivity $B_{p_{1 i}}$ of the $p_{1 i}$ is.

(2) Calculate the sensitivity $B_{q_{1 i}}$ of $q_{1 i}$

$$
B_{q_{1 i}}=\left(\frac{\partial K}{\partial_{q_{1 i}}}\right) \times \frac{q_{1 i}}{K} \approx \frac{p_{1 i} \times q_{1 i}}{\sum_{i=1}^{n} p_{1 i} \times q_{1 i}} \text { (The specific calculation process is the same as above. ) }
$$


As can be seen from the sensitivity of the expression of $q_{1 i}, B_{q_{1 i}}$ and $B_{p_{1 i}}$ are the same expression, indicating that the sensitivity of these two factors is the same.

(3) Calculate the sensitivity $B p_{0 i}$ of $p_{0 i}$

$$
B_{p_{0 i}}=\left(\frac{\partial K}{\partial p_{0 i}}\right) \times \frac{p_{0 i}}{K} \approx-\frac{10000 q_{0 i} \times \sum_{i=1}^{n} p_{1 i} q_{1 i}}{C P I\left(\sum_{i=1}^{n} p_{0 i} q_{01}\right)^{2}} \times \frac{p_{0 i}}{10000 \sum_{i=1}^{n} p_{1 i} q_{1 i} / C P I\left(\sum_{i=1}^{n} p_{0 i} q_{0 \mathrm{i}}\right)} \approx-\frac{p_{0 i} \times q_{0 \mathrm{i}}}{\sum_{i=1}^{n} p_{0 i} q_{0 \mathrm{i}}} .
$$

By the sensitivity expression of $p_{0 i}$ can be seen, $B p_{0 i}$ depends on $p_{0 i}$ and $q_{0 i}$. The bigger $p_{0 i} \times q_{0 i}$ is, the farmers' tobacco production cost index is more sensitive to $p_{0 i}$. While the smaller $\sum_{i=1}^{n} p_{0 i} q_{0 i}$ is, the farmers' tobacco production cost index is more sensitive to $p_{0 i}$. But $B p_{0 i}$ is a negative value. It indicates that the change direction of $p_{0 i}$ is opposite to the change of farmers' tobacco production cost index.

(4) Calculate the sensitivity $B_{q_{0 i}}$ of $q_{0 i}$

$$
B_{q_{0 i}}=\left(\frac{\partial K}{\partial q_{0 i}}\right) \times \frac{q_{0 i}}{K} \approx-\frac{p_{0 i} \times q_{0 i}}{\sum_{i=1}^{n} p_{0 i} q_{0 i}} \text { (The specific calculation process is the same as above.). }
$$

As can be seen from the sensitivity of the expression $q_{0 i}, B q_{0 i}$ and $B p_{0 i}$ has the same expression, indicating that the sensitivity of these two factors is the same.

Calculate the sensitivity of each variable.Based on the farmers' tobacco production cost index of a large professional at planting stage ${ }^{1}$, the sensitivity analysis of the influencing factors was carried out. $\quad p_{11}$ represents the unit family labor price of land preparation during transplanting in the report period, $p_{12}$ represents the unit hireling price of land preparation during transplanting in the report period, $p_{13}$ represents the unit family labor price of ridging during transplanting in the report period, and $p_{14}$ represents the unit hireling price of ridging during transplanting in the report period. The essential cost value is shown in table 1 and table 2 .

Table1 Statistical Table of Production Cost of Flue-cured Tobacco in Planting Stage

\begin{tabular}{|c|c|c|c|c|}
\hline Name & $P_{11}$ & $P_{12}$ & $P_{13}$ & $P_{14}$ \\
\hline Price (yuan) & 68 & 89 & 69 & 78 \\
\hline
\end{tabular}

Table2 Statistical Table of Production Cost of Flue-cured Tobacco in Planting Stage

\begin{tabular}{|c|c|c|c|c|c|}
\hline Name & $\mathrm{K}$ & $\partial \mathrm{K} / \partial \mathrm{P} 11$ & $\partial \mathrm{K} / \partial \mathrm{P} 12$ & $\partial \mathrm{K} / \partial \mathrm{P} 13$ & $\partial \mathrm{K} / \partial \mathrm{P} 14$ \\
\hline Value & 2602.25 & 0.15 & 0.14 & 0.19 & 0.38 \\
\hline
\end{tabular}

(1) Calculate the sensitivity of the unit family labor price of land preparation during transplanting in the report period [8].

1 Tobacco production is divided into four stages: seedling stage, transplanting stage, field management stage and classification stage sold baking. In this paper, the planting stage refers to the other three stages except the seedling stage. Flue-cured tobacco cultivation area of 20 acres to 100 acres of tobacco farmers is considered a large professional. 


$$
P_{p_{11}}=\left(\frac{\partial K}{\partial p_{11}}\right) \times \frac{p_{11}}{K} \approx \frac{p_{11} \times q_{11}}{\sum_{i=1}^{n} p_{1 i} q_{i}} \approx \frac{68 \times 0.15}{2602.25} \approx 0.0039
$$

Then the sensitivity of the professional large farms' tobacco production cost index at planting stage was 0.0039 for the unit family labor price of land preparation during transplanting in the report period; the unit family labor price of land preparation increases by one percentage points during transplanting in the report period, the professional large farms' tobacco production cost index will increase by 0.39 percentage points at planting stage.

(2) Calculate the sensitivity of the unit hireling price of land preparation during transplanting in the report period

$$
B_{p_{12}}=\left(\frac{\partial K}{\partial p_{12}}\right) \times \frac{p_{12}}{K} \approx \frac{p_{12} \times q_{12}}{\sum_{i=1}^{n} p_{1 i} q_{1 i}} \approx \frac{89 \times 0.14}{2602.25} \approx 0.0048
$$

Then the sensitivity of the professional large farms' tobacco production cost index at planting stage was 0.0048 for the unit hireling price of land preparation during transplanting in the report period; the unit hireling price of land preparation increases by one percentage points during transplanting in the report period, the professional large farms' tobacco production cost index will increase by 0.48 percentage points at planting stage.

(3) Calculate the sensitivity of the unit family labor price of ridging during transplanting in the report period

$$
B_{p_{13}}=\left(\frac{\partial K}{\partial p_{13}}\right) \times \frac{p_{13}}{K} \approx \frac{p_{13} \times q_{13}}{\sum_{i=1}^{n} p_{1 i} q_{1 i}} \approx \frac{69 \times 0.19}{2602.25} \approx 0.0050
$$

Then the sensitivity of the professional large farms' tobacco production cost index at planting stage was 0.0050 for the unit family labor price of ridging during transplanting in the report period; the unit family labor price of ridging increases by one percentage points during transplanting in the report period, the professional large farms' tobacco production cost index will increase by 0.50 percentage points at planting stage.

(4) Calculate the sensitivity of the unit hireling price of ridging during transplanting in the report period

$$
B_{p_{14}}=\left(\frac{\partial K}{\partial p_{14}}\right) \times \frac{p_{14}}{K} \approx \frac{p_{14} \times q_{14}}{\sum_{i=1}^{n} p_{1 i} q_{1 i}} \approx \frac{78 \times 0.38}{2602.25} \approx 0.0114
$$

Then the sensitivity of the professional large farms' tobacco production cost index at planting stage was 0.0114 for the unit hireling price of ridging during transplanting in the report period; the unit hireling price of ridging increases by one percentage points during transplanting in the report period, the professional large farms' tobacco production cost index will increase by 1.14 percentage points at planting stage.

Due to limited space, we only carried out a sensitivity analysis of the above four factors. According to the same method, we can calculate the sensitivity of the cost index to other factors, and sort the sensitive factors to find out the key factors to control the cost of tobacco production. 


\section{Conclusion}

In this paper, we introduce the related theory of sensitivity analysis and establish the sensitivity analysis model of the flue-cured tobacco production cost index. Then we take the production cost index of professional large tobacco production of Anshun City at the planting stage as a case study, and make a simple calculation and analysis to the sensitivity factors of the unit family labor price of land preparation during transplanting in the report period, the unit hireling price of land preparation during transplanting in the report period, the unit family labor price of ridging during transplanting in the report period and the unit hireling price of ridging during transplanting in the report period.

From the above four calculation results can be seen, the sensitive degree of the flue-cured tobacco production cost index to the four price from high to low in the planting stage is: the unit hireling price of ridging during transplanting in the report period, the unit family labor price of ridging during transplanting in the report period, the unit hireling price of land preparation during transplanting in the report period and the unit family labor price of land preparation during transplanting in the report period. Based on the above results, we can see the sensitivity of each factor to the flue-cured tobacco production cost index. We can also analyze the sensitivity of more factors influencing farmers' tobacco production cost index and sort the sensitivity factors for the preparation of cost control of the tobacco growing process.

\section{References}

[1] Kelei Yang, in: Technical Economics,edited by Fudan University Publising, Shanghai(2010) (In Chinese).

[2] Dongbing Huang, Guoqi Zhen: Guizhou Water Resources and Hydropower(2007) (In Chinese).

[3] Douglas L.Allaire, Karen E. Willcox: Distributional sensitivity analysis Procedia - Social and Behavioral Sciences (2010).

[4] Hu DW,Yuan JM: The Journal of Physical Chemistry (2006).

[5] Baoguang Liu, in: Sensitivity Data Analysis and Reliability Assessment, edited by National Defense Industry Publising, Beijing(1995) (In Chinese).

[6] Dongbing Huang, Datu Xu:Technical Economics(1994) (In Chinese).

[7] Jian Ge and Xiangchen Lei, in: Higher mathematics, edited by Science Press, Beijing:(2011) (In Chinese).

[8] Xiufeng Li,Yabin Wang : Chinese Journal of Mechanical Engineering(2014) (In Chinese). 